Research Article

\title{
Error Sensing Strategy for Active Control of Low-Frequency Sound Absorption of Micro-Perforated Panel Absorber by Using a Point Force Controlled Thin Plate
}

\author{
Xiyue Ma (D), Kean Chen, Lei Wang, and Yang Liu \\ School of Marine Science and Technology, Northwestern Polytechnical University, Xi'an 710072, China \\ Correspondence should be addressed to Xiyue Ma; xiyuema@nwpu.edu.cn
}

Received 12 December 2020; Revised 20 May 2021; Accepted 17 June 2021; Published 7 July 2021

Academic Editor: Giuseppe Ruta

Copyright (c) 2021 Xiyue Ma et al. This is an open access article distributed under the Creative Commons Attribution License, which permits unrestricted use, distribution, and reproduction in any medium, provided the original work is properly cited.

\begin{abstract}
This paper presents an analytical investigation on constructing an error sensing strategy of a new type of active MPPA. The proposed active MPPA is composed of MPP, air cavity, and point force-controlled backing panel, which can actively improve the low-frequency sound absorption of the MPPA. Constructing an appropriate error sensing strategy for obtaining an error signal that is highly correlated with the sound absorption coefficient of the active MPPA is a key problem encountered in practical implementation. The theoretical model of the active MPPA is firstly established using the modal analysis approach. Then, the active control performance and surface impedance characteristics in the controlled condition are analyzed in detail. Finally, the error sensing strategy of the active MPPA is constructed by measuring the surface average impedance ratio with an acoustic vector sensor (AVS). Simulation results show that, due to the antisymmetric property of the vibration of the backing panel on the resonant frequency, the surface impedance of the active MPPA after control also has symmetry or antisymmetry properties. Hence, the surface average impedance ratio of the active MPPA can be measured by using the limited number of acoustic vector sensors (sensing pressure and particle velocity). This variable is also highly correlated with the sound absorption coefficient of the active MPPA and thus can be used to construct the cost function (error signal). The active control result obtained by the proposed error sensing strategy is in good agreement with the theoretically optimal result, which validates the feasibility of this approach.
\end{abstract}

\section{Introduction}

The micro-perforated panel (MPP) is an innovative fiberfree solution to the noise control problem, which is widely used in areas such as room acoustics, environmental noise abatement, and duct mufflers [1]. By reducing the size of the orifices to the submillimeter scale, the MPP can provide enough acoustic resistance and low acoustic reactance [2]. The MPP absorber (MPPA) is the most commonly used form in practice in which the MPP is located in front of a rigid backing wall. MPP backed by a shallow air cavity can generate resonance. This increases the particle velocity through the holes, which can substantially dissipate the acoustical energy [2].

Generally, the MPPA is a wide-band absorber. The effective absorption band (sound absorption coefficient greater than 0.5) is a frequency band centered on its resonant frequency. Such bandwidth can only be limited to a specific range for the MPPA with specific structural parameters. Hence, it is insufficient to meet the practical application requirements. In order to broaden the absorption bandwidth, many improved design approaches are proposed. One approach involved is adding additional MPP into the air cavity of the MPPA to constitute a multiple-layer absorber [3-5] or parallel arranging multiple MPPAs with different cavity depths to form the large area sound absorption layer $[6,7]$. Broader absorption bandwidth can be achieved by combining different effective absorption bandwidths together. Another approach involved is to improve the structure of the MPPA. The irregular-shaped cavity-backed MPPA $[8,9]$ is proposed to enhance the vibro-acoustic coupling effect and effectively broaden the absorption 
bandwidth. In addition, filling the cavity with anisotropic fibrous material [10], decreasing the MPP holes diameter and thickness [11], and composing the MPPA with metamaterials [12, 13] and Helmholtz resonance structure [14] can also broaden the sound absorption bandwidth.

The abovementioned works significantly improve the sound absorption of the MPPA in the mid-high-frequency range. There are also many requirements for the MPPA with preferable low-frequency sound absorption, such as designing acoustic liners to reduce fan noise. A straightforward approach for improving the low-frequency sound absorption is to deepen the backing cavity to shift sound absorption bandwidth to the low-frequency range. However, such way at the cost of increased cavity depth can hardly be applied in engineering due to the practical limited installation space. In order to further broaden the low-frequency absorption bandwidth, passive and active methods are developed. The passive method mainly introduces resonant structure to obtain the improvement of sound absorption in a narrow bandwidth centered on the resonant frequency, such as the MPPA backed by Helmholtz resonators [15], the flexible micro-perforated panel absorber based on polyvinylidene fluoride piezoelectric film [16], the MPPA backed by shunted loudspeaker [17], and the MPPA backed with mechanical impedance plates [18].

On the contrary, the active control method can substantially broaden the low-frequency bandwidth. For a one-dimensional duct with plane wave excitation, an active control system in which the back wall is replaced by a piston source is proposed to actively control the lowfrequency sound absorption [19-21]. Two error sensing strategies are developed, i.e., impedance matching (IM) and pressure release (PR) strategies [19]. The IM strategy adjusts the velocity of the piston so as to maximally absorb the reflected plane wave in the cavity of the MPPA. Then, the specific acoustic impedance on the cavity side of the MPP will be close to that of the air medium, which indirectly adjusts the surface impedance of the MPPA to match that of the air medium. The PR strategy adjusts the velocity of the piston to an appropriate value so that the incident plane wave can be canceled by the reflected plane wave in the cavity. The sound impedance on the cavity side of the MPP is close to zero, which also indirectly adjusts the surface impedance of the MPPA to match that of the air medium.

The cone loudspeaker is usually used as the piston-type source in the above research. Then, the active MPPA will become jumbled and difficult to realize in engineering. On the contrary, if the ideal piston-type source is replaced by a secondary force-controlled elastic plate, the active MPPA will become easier to implement. The piezoelectric excitation or the small shaker placed in the cavity can be used as the control force in practice. The surface impedance of the active MPPA can also be adjusted to match the characteristic impedance of the air medium by actively controlling the vibration of the elastic plate. However, due to the complex vibration of the backing panel, the sound field in the cavity will become complex and can be no longer treated as the simplified superposition of the incident and reflected plane wave. Thus, the IM and PR strategy is not suitable to construct the error sensing strategy of such proposed active MPPA. Hence, the purpose of this paper is to fill in this gap. The vibration of the backing panel highly affects the surface impedance of the active MPPA. Due to the antisymmetric properties of the vibration of the backing panel on the resonant frequency, the surface impedance of the active MPPA also presents the symmetric or antisymmetric properties in controlled conditions. Thus, the surface average impedance of the active MPPA can be roughly predicted by using a limited number of acoustic vector sensors (sensing sound pressure and particle velocity) placed on the surface of the active MPPA. This variable is highly correlated with the sound absorption coefficient and can be used to construct the error sensing strategy of such active MPPA.

The remainder of this paper is organized as follows. The theoretical model is described in Section 2. The active control result and surface impedance of the proposed active MPPA are analyzed in Section 3. The error sensing strategy is constructed in Section 4. Conclusions are summarized in Section 5 .

\section{Theoretical Modeling}

2.1. Vibro-Acoustic Response of the Active MPPA. Figure 1 presents the side view of the proposed active MPPA. Ideal point force is applied in the research for simplification. The rectangular active MPPA is in the end of a rectangular duct so as to simplify the model. The cavity sound field can be adjusted by controlling the vibration of the backing panel with the point force, which indirectly adjusts the surface impedance of the active MPPA and improves the low-frequency sound absorption.

The sound velocity potential in the cavity is $\Phi(x, y, z, t)$, which satisfies the homogeneous wave equation [22]:

$$
\nabla^{2} \Phi(x, y, z, t)-\frac{1}{c_{0}^{2}} \frac{\partial^{2} \Phi(x, y, z, t)}{\partial t^{2}}=0
$$

where $c_{0}$ is the sound speed in air. The air particle velocity and the sound pressure in the cavity have the following relations with the sound velocity potential $\Phi(x, y, z, t)$ :

$$
\begin{aligned}
v_{x} & =\frac{\partial \Phi(x, y, z, t)}{\partial x}, \\
v_{y} & =\frac{\partial \Phi(x, y, z, t)}{\partial y}, \\
v_{z} & =\frac{\partial \Phi(x, y, z, t)}{\partial z}, \\
p(x, y, z, t) & =-\rho_{0} \frac{\partial \Phi(x, y, z, t)}{\partial t},
\end{aligned}
$$

where $v_{x}, v_{y}$, and $v_{z}$ are the air particle velocity along the $x, y$, and $z$ axes. $p(x, y, z, t)$ is the sound pressure in the cavity. $\rho_{0}$ is the density of air. The particle velocities on the surface of the MPP and the backing panel in the cavity satisfy the continuous boundary conditions: 


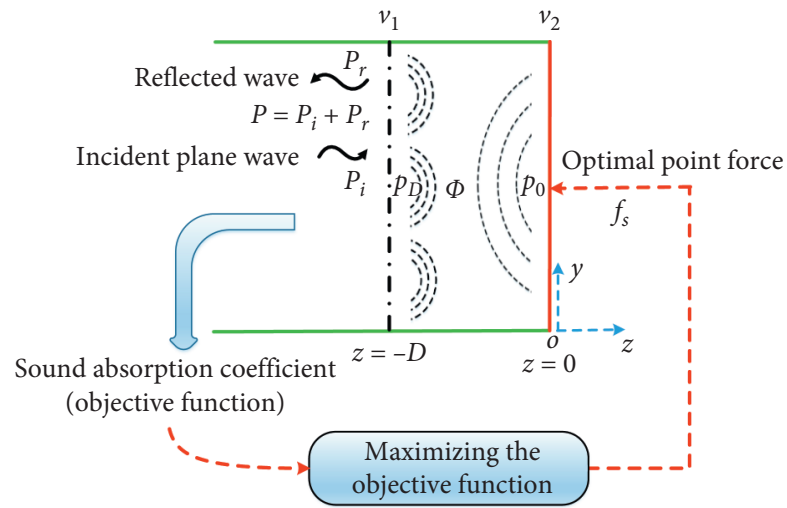

Figure 1: Side view of the proposed active MPPA.

$$
\begin{gathered}
\left.\frac{\partial \Phi(x, y, z, t)}{\partial z}\right|_{z=0}=v_{2}(x, y, t), \\
\left.\frac{\partial \Phi(x, y, z, t)}{\partial z}\right|_{z=-D}=v_{1}(x, y, t) .
\end{gathered}
$$

The boundary conditions on the other four rigid walls of the rectangular cavity are

$$
\begin{aligned}
& \left.\frac{\partial \Phi(x, y, z, t)}{\partial x}\right|_{x=0}=\left.\frac{\partial \Phi(x, y, z, t)}{\partial x}\right|_{x=a}=0, \\
& \left.\frac{\partial \Phi(x, y, z, t)}{\partial y}\right|_{y=0}=\left.\frac{\partial \Phi(x, y, z, t)}{\partial y}\right|_{y=b}=0,
\end{aligned}
$$

where $v_{1}(x, y, t)$ and $v_{2}(x, y, t)$ in equations (6) and (7) are the surface velocity of the MPP and the backing panel, respectively. $a$ and $b$ are the length and width of the MPP. $D$ is the depth of the cavity. Assuming that the distance between the MPP holes is much lower than the acoustic wavelength, $v_{1}(x, y, t)$ can be considered as a distributed particle velocity, spatially averaged over each aperture cell adjacent to the holes [23]:

$$
v_{1}(x, y, t) \approx v_{M}(x, y)+\sigma \frac{P-P_{D}}{z_{0}},
$$

where $v_{M}(x, y)$ is the velocity of the MPP, and $\sigma$ is the perforation ratio of the MPP. $P$ is the total sound pressure on the incident side, $P_{D}(x, y)$ is the sound pressure at $z=-D$ in the cavity. The total sound pressure loading can be written as the sum of incident pressure $P_{i}$, reflected pressure $P_{r}$, and the pressure $P_{\text {rad }}$ back radiated by the elastic MPP, $P=P_{i}+P_{r}+P_{\text {rad }}$. The incident plane wave can be expressed as $P_{i}=p_{0} e^{j\left(\omega t-k_{0} z\right)} \cdot p_{0}$ is the amplitude of the incident plane wave, and $k_{0}=\omega / c_{0}$ is the wavenumber. $P_{\text {rad }}$ can be negligible as long as the back-radiated sound power is a small fraction of the power dissipated through the structure and the holes. $z_{0}$ is the impedance of the MPP, which can be expressed as [1]

$$
\begin{aligned}
z_{0}= & \frac{32 \eta h_{M}}{d^{2}}\left[\sqrt{1+\frac{k_{h}^{2}}{32}}+\frac{\sqrt{2}}{32} k_{h} \frac{d}{h_{M}}\right] \\
& +j \rho_{0} \omega h_{M}\left[1+\left(9+\frac{k_{h}^{2}}{2}\right)^{-(1 / 2)}+\frac{8}{3 \pi} \frac{d}{h_{M}}\right],
\end{aligned}
$$

where $d$ is the diameter of the hole, $h_{M}$ is the panel thickness, $\eta$ is the coefficient of viscosity of the air, $k_{h}$ is the perforate constant $k_{h}=(d / 2) / r_{\text {visc }}(\omega)$, and $r_{\text {visc }}(\omega)$ is the viscous boundary layer thickness $r_{\text {visc }}(\omega)=\sqrt{\eta / \rho_{0} \omega}$.

According to equations (8) and (9), the solution of equation (1), i.e., sound velocity potential $\Phi(x, y, z, t)$, can be expressed as [23]

$$
\begin{aligned}
\Phi(x, y, z, t)= & \sum_{u=0}^{U} \sum_{w=0}^{W}\left[L^{u w} \cos h\left(\mu^{u w} z\right)\right. \\
& \left.+N^{u w} \sin h\left(\mu^{u w} z\right)\right] \cos \left(\frac{u \pi x}{a}\right) \cos \left(\frac{w \pi y}{b}\right) e^{j \omega t},
\end{aligned}
$$

where

$$
\mu^{u w}=\sqrt{\left(\frac{u \pi}{a}\right)^{2}+\left(\frac{w \pi}{b}\right)^{2}+\left(\frac{\omega}{c_{0}}\right)^{2}},
$$

$\omega$ is the angular frequency, $U$ and $W$ are the numbers of the acoustic modes, and $(u, w)$ is the modal index in the $(x, y)$ plane. The unknown coefficient $L^{u w}$ and $N^{u w}$ in equation (12) can be derived by applying the boundary conditions of equations (6) and (7). Substituting equation (12) into equation (6), together with the orthogonality of the modal shape function, $N^{u w}$ can be derived and expressed as (the harmonic time factor $e^{\mathrm{j} \omega t}$ is omitted)

$$
N^{u w}=\frac{\int_{0}^{a} \int_{0}^{b} v_{2}(x, y) \Psi_{u w}(x, y) \mathrm{d} x \mathrm{~d} y}{\mu^{u w} \beta^{u w}},
$$

where $\Psi_{u w}(x, y)=\cos (u \pi x / a) \cos (w \pi y / b), \quad$ and $\beta^{u w}=\int_{0}^{a} \int_{0}^{b} \cos ^{2}(u \pi x / a) \cos ^{2}(w \pi y / b) \mathrm{d} x \mathrm{~d} y$. In the same way, the coefficient $L^{u w}$ can be derived and expressed as

$$
\begin{aligned}
L^{u w}= & \frac{\cosh \left(D \mu^{u w}\right) \int_{0}^{a} \int_{0}^{b} v_{2}(x, y) \Psi_{u w}(x, y) \mathrm{d} x \mathrm{~d} y}{\mu^{u w} \beta^{u w} \sin h\left(D \mu^{u w}\right)} \\
& -\frac{\int_{0}^{a} \int_{0}^{b}\left[v_{M}(x, y)+\sigma\left(P-P_{D}\right) / z_{0}\right] \Psi_{u w}(x, y) \mathrm{d} x \mathrm{~d} y}{\mu^{u w} \beta^{u w} \sin h\left(D \mu^{u w}\right)} .
\end{aligned}
$$

According to equation (5), the sound pressure on the surface of the MPP in the cavity side $(z=-D)$ can be expressed as 


$$
\begin{aligned}
P_{D}= & -j \omega \rho_{0} \sum_{u=0}^{U} \sum_{w=0}^{W}\left[L^{u w} \cos h\left(-D \mu^{u w}\right)\right. \\
& \left.+N^{u w} \sin h\left(D \mu^{u w}\right)\right] \Psi_{u w}(x, y) .
\end{aligned}
$$

Substituting equation (14) and (15) into equation (16), the sound pressure $P_{D}$ can be expressed as

$$
\begin{aligned}
P_{D}= & \sum_{u=0}^{U} \sum_{w=0}^{W} \frac{z_{u w}^{t}}{\beta^{u w}}\left\{\int_{0}^{a} \int_{0}^{b}\left[v_{M}(x, y)+\sigma \frac{P-P_{D}}{z_{0}}\right] \Psi_{u w}(x, y) \mathrm{d} x \mathrm{~d} y\right\} \Psi_{u w}(x, y) \\
& -\sum_{u=0}^{U} \sum_{w=0}^{W} \frac{z_{u w}^{s}}{\beta^{u w}}\left[\int_{0}^{a} \int_{0}^{b} v_{2}(x, y) \Psi_{u w}(x, y) \mathrm{d} x \mathrm{~d} y\right] \Psi_{u w}(x, y),
\end{aligned}
$$

where $z_{u w}^{t}=j \omega \rho_{0} /\left(\mu^{u w} \tan h\left(D \mu^{u w}\right)\right)$, and $z_{u w}^{s}=j \omega \rho_{0} /\left(\mu^{u w}\right.$ $\left.\sin h\left(D \mu^{u w}\right)\right)$. In the same way, the sound pressure $P_{0}$ on

the surface of the backing panel $(z=0)$ can be expressed as

$$
\begin{aligned}
P_{0}= & \sum_{u=0}^{U} \sum_{w=0}^{W} \frac{z_{u w}^{s}}{\beta^{u w}}\left\{\int_{0}^{a} \int_{0}^{b}\left[v_{M}(x, y)+\sigma \frac{P-P_{D}}{z_{0}}\right] \Psi_{u w}(x, y) \mathrm{d} x \mathrm{~d} y\right\} \Psi_{u w}(x, y) \\
& -\sum_{u=0}^{U} \sum_{w=0}^{W} \frac{z_{u w}^{t}}{\beta^{u w}}\left[\int_{0}^{a} \int_{0}^{b} v_{2}(x, y) \Psi_{u w}(x, y) \mathrm{d} x \mathrm{~d} y\right] \Psi_{u w}(x, y) .
\end{aligned}
$$

In equation (17), the sound pressure $P_{D}$ is expressed in terms of itself. Hence, equation (17) should be rearranged. Multiplying $\Psi_{u w}(x, y)$ on both sides of equation (17), and

using the orthogonality of modal shape function, the following expression can be obtained:

$$
\begin{aligned}
\int_{0}^{a} \int_{0}^{b} P_{D} \Psi_{u w}(x, y) \mathrm{d} x \mathrm{~d} y= & \frac{z_{0} z_{u w}^{t}}{z_{0}+\sigma z_{u w}^{t}} \int_{0}^{a} \int_{0}^{b}\left[v_{M}(x, y)+\sigma \frac{P}{z_{0}}\right] \Psi_{u w}(x, y) \mathrm{d} x \mathrm{~d} y \\
& -\frac{z_{0} z_{u w}^{s}}{z_{0}+\sigma z_{u w}^{t}} \int_{0}^{a} \int_{0}^{b} v_{2}(x, y) \Psi_{u w}(x, y) \mathrm{d} x \mathrm{~d} y .
\end{aligned}
$$

Substituting equation (19) into equation (17) yields the expression of sound pressure $P_{D}$ as

$$
\begin{aligned}
P_{D}= & \sum_{u=0}^{U} \sum_{w=0}^{W} G_{u w}\left\{\int_{0}^{a} \int_{0}^{b}\left[v_{M}(x, y)+\sigma \frac{P}{z_{0}}\right] \Psi_{u w}(x, y) \mathrm{d} x \mathrm{~d} y\right\} \Psi_{u w}(x, y) \\
& -\sum_{u=0}^{U} \sum_{w=0}^{W} H_{u w}\left[\int_{0}^{a} \int_{0}^{b} v_{2}(x, y) \Psi_{u w}(x, y) \mathrm{d} x \mathrm{~d} y\right] \Psi_{u w}(x, y),
\end{aligned}
$$

where $G_{u w}=z_{0} z_{u w}^{t} /\left(\beta^{u w}\left(z_{0}+\sigma z_{u w}^{t}\right)\right)$, and $H_{u w}=z_{0} z_{u w}^{s} /$ $\left(\beta^{u w}\left(z_{0}+\sigma z_{u w}^{t}\right)\right)$. Then, substituting equation (19) into equation (18) yields the expression of sound pressure $P_{0}$ as 


$$
\begin{aligned}
P_{0}= & \sum_{u=0}^{U} \sum_{w=0}^{W} H_{u w}\left\{\int_{0}^{a} \int_{0}^{b}\left[v_{M}(x, y)+\sigma \frac{P}{z_{0}}\right] \Psi_{u w}(x, y) \mathrm{d} x \mathrm{~d} y\right\} \Psi_{u w}(x, y) \\
& +\sum_{u=0}^{U} \sum_{w=0}^{W} I_{u w}\left[\int_{0}^{a} \int_{0}^{b} v_{2}(x, y) \Psi_{u w}(x, y) \mathrm{d} x \mathrm{~d} y \Psi_{u w}(x, y)\right],
\end{aligned}
$$

where $I_{u w}=\left(\sigma\left(z_{u w}^{s}\right)^{2}-z_{0} z_{u w}^{t}-\sigma\left(z_{u w}^{t}\right)^{2}\right) /\left(\beta^{u w}\left(z_{0}+\sigma z_{u w}^{t}\right)\right)$.

The sound energy absorbed by the active MPPA is a function of the control force. The reflected wave and the total sound pressure $P$ on the surface of the active MPPA also change as the point force changes. The acoustic velocity potential of the reflected wave at the incident side is $\Phi_{r}(x, y, z, \omega)$, which can be expressed as [6]

$$
\Phi_{r}(x, y, z, \omega)=\sum_{u=0}^{U} \sum_{w=0}^{W} R_{u w} e^{\mu^{u \omega}} \Psi_{u w}(x, y) .
$$

Then, the total acoustic velocity potential $\Phi_{L}=\Phi_{i}+\Phi_{r}$ on the incident side $(z=-D)$ satisfies the following continuity boundary condition:

$$
\left.\left(\frac{\partial \Phi_{i}}{\partial z}+\frac{\partial \Phi_{r}}{\partial z}\right)\right|_{z=-D}=v_{1}(x, y)
$$

where $\Phi_{i}$ is sound velocity potential of the incident plane wave, $\Phi_{i}=-\left(p_{0} / j \omega \rho_{0}\right) e^{-j k_{0} z}$. Substituting equation (22) into equation (23) yields the equation that the unknown coefficient $R_{u w}$ satisfies:

$$
\frac{p_{0} k_{0}}{\omega \rho_{0}} e^{j k_{0} D}+\sum_{u=0}^{U} \sum_{w=0}^{W} R_{u w} \mu^{u w} e^{-\mu^{u w} D} \Psi_{u w}(x, y)=v_{1}(x, y) .
$$

Multiplying $\Psi_{u w}(x, y)$ on both sides of equation (24) and applying the orthogonality of the modal shape function yield the expression of $R_{u w}$ :

$$
\begin{aligned}
R_{u w}= & \frac{\int_{0}^{a} \int_{0}^{b} v_{1}(x, y) \Psi_{u w}(x, y) \mathrm{d} x \mathrm{~d} y}{\mu^{u w} e^{-\mu^{u w} D} \beta^{u w}} \\
& -\frac{p_{0} k_{0} e^{j k_{0} D} \int_{0}^{a} \int_{0}^{b} \Psi_{u w}(x, y) \mathrm{d} x \mathrm{~d} y}{\omega \rho_{0} \mu^{u w} e^{-\mu^{u w} D} \beta^{u w}} .
\end{aligned}
$$

Substituting equations (22) and (25) into equation (5), the sound pressure $P_{r}$ of the reflected wave at the surface of the active MPPA $(z=-D)$ can be expressed as

$$
\begin{aligned}
P_{r}= & \sum_{u=0}^{U} \sum_{w=0}^{W} \frac{Z_{u w}^{r}}{\beta^{u w}}\left\{\int_{0}^{a} \int_{0}^{b}\left[v_{M}(x, y)+\sigma \frac{P}{z_{0}}\right] \Psi_{u w}(x, y) \mathrm{d} x \mathrm{~d} y\right\} \Psi_{u w}(x, y) \\
& -\sum_{u=0}^{U} \sum_{w=0}^{W} \frac{Z_{u w}^{r}}{\beta^{u w}}\left[\int_{0}^{a} \int_{0}^{b} \sigma \frac{P_{D}}{z_{0}} \Psi_{u w}(x, y) \mathrm{d} x \mathrm{~d} y\right] \Psi_{u w}(x, y)+\frac{j p_{0} k_{0} a b e^{j k_{0} D}}{\mu^{00} \beta^{00}}
\end{aligned}
$$

where $Z_{u w}^{r}=-j \omega \rho_{0} / \mu^{u w}$. Further substituting equation (19) into equation (26), the reflected sound pressure $P_{r}$ can be expressed as

$$
\begin{aligned}
P_{r}= & \sum_{u=0}^{U} \sum_{w=0}^{W} J_{u w}\left\{\int_{0}^{a} \int_{0}^{b}\left[v_{M}(x, y)+\sigma \frac{P}{z_{0}}\right] \Psi_{u w}(x, y) \mathrm{d} x \mathrm{~d} y\right\} \Psi_{u w}(x, y) \\
& +\sum_{u=0}^{U} \sum_{w=0}^{W} K_{u w}\left[\int_{0}^{a} \int_{0}^{b} v_{2}(x, y) \Psi_{u w}(x, y) \mathrm{d} x \mathrm{~d} y\right] \Psi_{u w}(x, y)+\frac{j p_{0} k_{0} a b e^{j k_{0} D}}{\mu^{00} \beta^{00}}
\end{aligned}
$$

where $J_{u w}=z_{0} Z_{u w}^{r} /\left(\beta^{u w}\left(z_{0}+\sigma Z_{u w}^{t}\right)\right)$, and $K_{u w}=z_{0} Z_{u w}^{r}$ $Z_{u w}^{s} /\left(\beta^{u w}\left(z_{0}+\sigma Z_{u w}^{t}\right)\right)$. The right side of equation (27) also contains the reflected sound pressure $P_{r}$, i.e., $P=P_{i}+P_{r}$. Thus, equation (27) should be further rearranged. Multiplying $\Psi_{u w}(x, y)$ on both sides of the equation (27) and applying the orthogonality of the modal shape function yield the expression 


$$
\begin{aligned}
\int_{0}^{a} \int_{0}^{b} P_{r} \Psi_{u w}(x, y) \mathrm{d} x \mathrm{~d} y= & \frac{\left(z_{0}+\sigma Z_{u w}^{t}\right) J_{u w} \beta^{u w}}{z_{0}+\sigma Z_{u w}^{t}-\sigma Z_{u w}^{r}}\left[\int_{0}^{a} \int_{0}^{b} v_{M}(x, y) \Psi_{u w}(x, y) \mathrm{d} x \mathrm{~d} y\right] \\
& +\frac{\left(z_{0}+\sigma Z_{u w}^{t}\right) K_{u w} \beta^{u w}}{z_{0}+\sigma Z_{u w}^{t}-\sigma Z_{u w}^{r}}\left[\int_{0}^{a} \int_{0}^{b} v_{2}(x, y) \Psi_{u w}(x, y) \mathrm{d} x \mathrm{~d} y\right] \\
& +\left(\frac{J_{00} \sigma P_{i} a b}{z_{0}}+\frac{j p_{0} k_{0} a b e^{j k_{0} D}}{\mu^{00} \beta^{00}}\right) \frac{z_{0}+\sigma Z_{u w}^{t}}{z_{0}+\sigma Z_{u w}^{t}-\sigma Z_{u w}^{r}}\left[\int_{0}^{a} \int_{0}^{b} \Psi_{u w}(x, y) \mathrm{d} x \mathrm{~d} y\right]
\end{aligned}
$$

Substituting equation (28) into equation (27) yields the reflected sound pressure $P_{r}$ as

$$
\begin{aligned}
P_{r}= & \sum_{u=0}^{U} \sum_{w=0}^{W} E_{u w}\left[\int_{0}^{a} \int_{0}^{b} v_{M}(x, y) \Psi_{u w}(x, y) \mathrm{d} x \mathrm{~d} y\right] \Psi_{u w}(x, y) \\
& +\sum_{u=0}^{U} \sum_{w=0}^{W} F_{u w}\left[\int_{0}^{a} \int_{0}^{b} v_{2}(x, y) \Psi_{u w}(x, y) \mathrm{d} x \mathrm{~d} y\right] \Psi_{u w}(x, y)+D_{00}
\end{aligned}
$$

where $E_{u w}=\left(\left(z_{0}+\sigma Z_{u w}^{t}\right) /\left(z_{0}+\sigma Z_{u w}^{t}-\sigma Z_{u w}^{r}\right)\right) J_{u w}, F_{u w}=$ $J_{u w} \quad \sigma^{2} Z_{u w}^{r} Z_{u w}^{s} /\left(z_{0}\left(z_{0}+\sigma Z_{u w}^{t}-\sigma Z_{u w}^{r}\right)\right)+K_{u w}$, and $D_{00}=$ $J_{00} \sigma \quad\left(P_{i} / z_{0}\right) a b+J_{00}\left(\sigma / z_{0}\right) a b\left(J_{00} \sigma\left(P_{i} / z_{0}\right) a b+\left(j p_{0} k_{0}\right.\right.$ $\left.\left.a b e^{\left(j k_{0} D\right)} /\left(\mu^{00} \beta^{00}\right)\right)\right)\left(z_{0}+\sigma Z_{00}^{t}\right) /\left(z_{0}+\sigma Z_{00}^{t}-\sigma Z_{00}^{r}\right)+\left(j p_{0}\right.$ $\left.k_{0} a b e^{\left(j k_{0} D\right)} /\left(\mu^{00} \beta^{00}\right)\right)$.

As for the elastic MPP and backing panel, their displacements can be expressed as the superposition of a series of modes:

$$
\begin{aligned}
& w_{M}(x, y, t)=\sum_{m=1}^{M} \sum_{n=1}^{N} A_{m n} \phi_{m}(x) \varphi_{n}(y), \\
& w_{2}(x, y, t)=\sum_{m=1}^{M} \sum_{n=1}^{N} B_{m n} \phi_{m}(x) \varphi_{n}(y),
\end{aligned}
$$

where $w_{M}(x, y, t)$ and $w_{2}(x, y, t)$ are the displacements of the MPP and backing panel, $A_{m n}$ and $B_{m n}$ are the modal amplitudes of the $(m, n)$ th mode. $\phi_{m}(x) \varphi_{n}(y)$ is the modal shape function and can be expressed as $\phi_{m}(x)$ $\varphi_{n}(y)=\sin (m \pi x / a) \sin (n \pi y / b)$ for simply supported boundary condition. Under the excitation of the total sound pressure $P$ and the sound pressure $P_{D}$ in the cavity, the displacement of the MPP satisfies the wave equation:

$$
D_{M}\left(\frac{\partial^{4} w_{M}}{\partial x^{4}}+2 \frac{\partial^{4} w_{M}}{\partial x^{2} \partial y^{2}}+\frac{\partial^{4} w_{M}}{\partial y^{4}}\right)+\rho_{M} h_{M} \frac{\partial^{2} w_{M}}{\partial t^{2}}=P-P_{D}
$$

where $D_{M}, \rho_{M}$, and $h_{M}$ are the bending stiffness, density, and thickness of the MPP, respectively. Substituting equation (30) into equation (32), multiplying $\phi_{m}(x) \varphi_{n}(y)$ on both sides of the equation (32), and applying the orthogonality of the modal shape function yield the equation that the modal amplitude $A_{m n}$ satisfies:

$$
A_{m n} \alpha_{m n} Z_{m n}=\int_{0}^{a} \int_{0}^{b}\left(P-P_{D}\right) \sin \left(\frac{m \pi x}{a}\right) \sin \left(\frac{n \pi y}{b}\right) \mathrm{d} x \mathrm{~d} y
$$

where the coefficient $\alpha_{m n}=\int_{0}^{a} \int_{0}^{b} \sin ^{2} \quad(m \pi x / a) \sin ^{2}$ $(n \pi y / b) \mathrm{d} x \mathrm{~d} y=a b / 4$, and $Z_{m n}=\rho_{M} h_{M}\left(\omega_{m n}^{2}+j \xi_{m n} \omega_{m n}\right.$ $\left.\omega-\omega^{2}\right) \cdot \omega_{m n}$ and $\xi_{m n}$ are the resonant frequency and damping ratio of the $(m, n)$ th mode, respectively. Substituting (20) and (29) into (33) together with (28) yields the equation that the modal amplitude $A_{m n}$ satisfies:

$$
\begin{aligned}
A_{m n} \alpha_{m n} Z_{m n}= & \sum_{u=0}^{U} \sum_{w=0}^{W} S_{u w}\left[\int_{0}^{a} \int_{0}^{b} v_{M}(x, y) \Psi_{u w}(x, y) \mathrm{d} x \mathrm{~d} y\right] \gamma_{m n}^{u w} \\
& +\sum_{u=0}^{U} \sum_{w=0}^{W} T_{u w}\left[\int_{0}^{a} \int_{0}^{b} v_{2}(x, y) \Psi_{u w}(x, y) \mathrm{d} x \mathrm{~d} y\right] \gamma_{m n}^{u w}+V_{m n},
\end{aligned}
$$

where the coefficient $\gamma_{m n}^{u w}=\int_{0}^{a} \int_{0}^{b} \cos (u \pi x / a) \cos (w \pi$ $y / b) \sin (m \pi x / a) \sin (n \pi y / b) \mathrm{d} x \mathrm{~d} y$.
The coefficients $S_{u w}, T_{u w}$, and $V_{m n}$ in (34) can be expressed as $S_{u w}=E_{u w}-G_{u w}-\sigma Z_{u w}^{r} G_{u w} /\left(z_{0}+\sigma Z_{u w}^{t}\right.$ 
$\left.-\sigma Z_{u w}^{r}\right), \quad T_{u w}=F_{u w}-\sigma^{2} G_{u w} Z_{u w}^{r} Z_{u w}^{s} /\left(z_{0}\left(z_{0}+\sigma Z_{u w}^{t}\right.\right.$ $\left.\left.-\sigma Z_{u w}^{r}\right)\right)+H_{u w}$, and $V_{m n}=P_{i} \xi_{m n}+D_{00} \xi_{m n}-G_{00} \sigma\left(P_{i} /\right.$ $\left.z_{0}\right) a b \xi_{m n}-G_{00}\left(\sigma / z_{0}\right)\left(J_{00} \sigma \quad\left(P / z_{0}\right) a b+\left(j p_{0} k_{0} a b e\right)^{\left(j k_{0} D\right)} /\right.$ $\left.\left.\left(\mu^{00} \beta^{00}\right)\right)\right) \quad\left(\left(z_{0}+\sigma Z_{00}^{t}\right) /\left(z_{0}+\sigma Z_{00}^{t}-\sigma Z_{00}^{r}\right)\right) a b \xi_{m n}$, where $\xi_{m n}=\int_{0}^{a} \int_{0}^{b} \sin (m \pi x / a) \sin (n \pi y / b) \mathrm{d} x \mathrm{~d} y$. Further substituting (30) and (31) into (34) yields the equation that the modal amplitudes $A_{m n}$ and $B_{m n}$ satisfy:

$$
\begin{gathered}
A_{m n} \alpha_{m n} Z_{m n}-\sum_{m^{\prime}=1}^{M} \sum_{n^{\prime}=1}^{N} A_{m^{\prime} n^{\prime}}\left[\sum_{u=0}^{U} \sum_{w=0}^{W} j \omega S_{u w} \gamma_{u w}^{m^{\prime \prime} n} \gamma_{m n}^{u w}\right] \\
-\sum_{m^{\prime}=1}^{M} \sum_{n^{\prime}=1}^{N} B_{m^{\prime} n^{\prime}}\left[\sum_{u=0}^{U} \sum_{w=0}^{W} j \omega T_{u w} \gamma_{u w}^{m^{\prime} n} \gamma_{m n}^{u w}\right]=V_{m n} .
\end{gathered}
$$

If the following variables are further defined $C_{1}\left(m n, m^{\prime} n^{\prime}\right)=\alpha_{m n} Z_{m n} \delta\left(m-m^{\prime}\right) \delta \quad\left(n-n^{\prime}\right)-\sum_{u=0}^{U} \quad \sum_{w=0}^{W}$ $j \omega S_{u w} \gamma_{u w}^{m^{\prime} n^{\prime}} \gamma_{m n}^{u w}$, and $D_{1}\left(m n, m^{\prime} n^{\prime}\right)=-\sum_{u=0}^{U} \quad \sum_{w=0}^{W}$ $j \omega T_{u w} \gamma_{u w}^{m^{\prime} n^{\prime}} \gamma_{m n}^{u w}$, the $M \times N$ unknown modal amplitudes of the MPP and backing panel satisfy the matrix equation:

$$
\mathrm{C}_{1} \mathbf{A}+\mathrm{D}_{1} \mathbf{B}=\mathbf{V}
$$

where $\mathbf{A}=\left[A_{11}, \ldots, A_{m n}, \ldots, A_{M N}\right]^{T}, \mathbf{B}=\left[B_{11}, \ldots\right.$, $\left.B_{m n}, \ldots, B_{M N}\right]^{T}$, and $\mathbf{V}=\left[V_{11}, \ldots, V_{m n}, \ldots, V_{M N}\right]^{T}$.
For the backing panel, under the excitation of the sound pressure $P_{0}$ and the control force $f_{s}=F_{s} \delta\left(x-x_{s}, y-y_{s}\right)$, the displacement satisfies the following wave equation:

$$
D_{2}\left(\frac{\partial^{4} w_{2}}{\partial x^{4}}+2 \frac{\partial^{4} w_{2}}{\partial x^{2} \partial y^{2}}+\frac{\partial^{4} w_{2}}{\partial y^{4}}\right)+\rho_{2} h_{2} \frac{\partial^{2} w_{2}}{\partial t^{2}}=P_{0}+f_{s}
$$

where $D_{2}, \rho_{2}$, and $h_{2}$ are the bending stiffness, density, and thickness of the backing panel, respectively. Substituting (31) into (37) together with the orthogonality of the modal shape function yields the equation that the modal amplitude $B_{m n}$ satisfies:

$$
B_{m n} \alpha_{m n} Z_{2, m n}=\int_{0}^{a} \int_{0}^{b} P_{0} \sin \left(\frac{m \pi x}{a}\right) \sin \left(\frac{n \pi y}{b}\right) \mathrm{d} x \mathrm{~d} y+F_{s} Q_{s, m n},
$$

where $Z_{2, m n}=\rho_{2} h_{2}\left(\omega_{2, m n}^{2}+j \xi_{2, m n} \omega_{2, m n} \omega-\omega^{2}\right), \omega_{2, m n}$ and $\xi_{2, m n}$ are the resonant frequency and the damping ratio of the $(m, n)$ th mode of the backing panel, $F_{s}$ is the amplitude of the control force, and $Q_{s, m n}$ is the generalized secondary modal force $Q_{s, m n}=\phi_{m}\left(x_{s}\right) \varphi_{n}\left(y_{s}\right)$. Further substituting (21) and (28) into (38) yields the equation that the modal amplitude $B_{m n}$ satisfies:

$$
\begin{aligned}
B_{m n} \alpha_{m n} Z_{P, m n}= & \sum_{u=0}^{U} \sum_{w=0}^{W} X_{u w}\left\{\int_{0}^{a} \int_{0}^{b} v_{M}(x, y) \Psi_{u w}(x, y) \mathrm{d} x \mathrm{~d} y\right\} \gamma_{m n}^{u w} \\
& +\sum_{u=0}^{U} \sum_{w=0}^{W} Y_{u w}\left[\int_{0}^{a} \int_{0}^{b} v_{2}(x, y) \Psi_{u w}(x, y) \mathrm{d} x \mathrm{~d} y\right] \gamma_{m n}^{u w}+V_{2, m n}+F_{s} Q_{s, m n},
\end{aligned}
$$

where the variables $X_{u w}, Y_{u w}$, and $V_{2, m n}$ can be expressed as $X_{u w}=H_{u w}+\sigma H_{u w} Z_{u w}^{r} /\left(z_{0}+\sigma Z_{u w}^{t}-\sigma Z_{u w}^{r}\right), Y_{u w}=\left(\sigma H_{u w}\right.$ $Z_{u w}^{r} Z_{u w}^{s} /\left(z_{0}\left(z_{0}+\sigma Z_{u w}^{t}-\sigma Z_{u w}^{r}\right)\right)+I_{u w}$, and $V_{2, m n}=H_{00}$ $\sigma\left(P_{i} / z_{0}\right) a b \xi_{m n}+H_{00}\left(\sigma / z_{0}\right)\left(J_{00} \sigma\left(P_{i} / z_{0}\right) a b+\quad\left(j p_{0} k_{0}\right.\right.$ $\left.\left.a b e)^{\left(j k_{0} D\right)} /\left(\mu^{00} \beta^{00}\right)\right)\right)\left(\left(z_{0}+\sigma Z_{00}^{t}\right) /\left(z_{0}+\sigma Z_{00}^{t}-\sigma Z_{00}^{r}\right)\right) a b \xi_{m n}$.

Substituting (30) and (31) into (39) yields the equation that the modal amplitudes $A_{m n}$ and $B_{m n}$ satisfy:

$$
\begin{aligned}
& B_{m n} \alpha_{m n} Z_{p, m n}-\sum_{m^{\prime}=1}^{M} \sum_{n^{\prime}=1}^{N} A_{m^{\prime} n^{\prime}}\left[\sum_{u=0}^{U} \sum_{w=0}^{W} j \omega X_{u w} \gamma_{u w}^{m^{\prime} n^{\prime}} \gamma_{m n}^{u w}\right] \\
& \quad-\sum_{m^{\prime}=1}^{M} \sum_{n^{\prime}=1}^{N} B_{m^{\prime} n^{\prime}}\left[\sum_{u=0}^{U} \sum_{w=0}^{W} j \omega Y_{u w} \gamma_{u w}^{m^{\prime} n^{\prime}} \gamma_{m n}^{u w}\right] \\
& =V_{2, m n}+F_{s} Q_{s, m n} .
\end{aligned}
$$

If the following variables are further defined, $C_{2}\left(m n, m^{\prime} n^{\prime}\right)=-\sum_{u=0}^{U} \sum_{w=0}^{W} j \omega X_{u w} \gamma_{u w}^{m^{\prime} n^{\prime}} \gamma_{m n}^{u w}, D_{2}\left(m n, m^{\prime} n^{\prime}\right)$ $=\alpha_{m n} Z_{p}, \quad m n \delta\left(m-m^{\prime}\right) \delta\left(n-n^{\prime}\right)-\sum_{u=0}^{U} \sum_{w=0}^{W} j \omega Y_{u w} \gamma_{u w}^{m^{\prime} n^{\prime}}$ $\gamma_{m n}^{u w}$, the $M \times N$ unknown modal amplitudes of the $A_{m n}$ and $B_{m n}$ satisfy the matrix equation:

$$
\mathbf{C}_{2} \mathbf{A}+\mathbf{D}_{2} \mathbf{B}=\mathbf{V}_{2}+F_{s} \mathbf{Q}_{s}
$$

where $\quad \mathbf{Q}_{s}=\left[\phi_{1}\left(x_{s}\right) \varphi_{1}\left(y_{s}\right), \phi_{1}\left(x_{s}\right) \varphi_{2}\left(y_{s}\right), \ldots, \phi_{M}\left(x_{s}\right)\right.$ $\left.\varphi_{N}\left(y_{s}\right)\right]^{T}$, and $\mathbf{V}_{2}=\left[V_{2,11}, \ldots, V_{2, m n}, \ldots, V_{2, M N}\right]^{T}$.

Combining (36) and (41), the modal amplitudes of the MPP and backing panel can be solved:

$$
\left[\begin{array}{l}
\mathbf{A} \\
\mathbf{B}
\end{array}\right]=\left[\begin{array}{ll}
\mathbf{C}_{1} & \mathbf{D}_{1} \\
\mathbf{C}_{2} & \mathbf{D}_{2}
\end{array}\right]^{-1}\left[\begin{array}{c}
\mathbf{V} \\
\mathbf{V}_{2}+F_{s} \mathbf{Q}_{s}
\end{array}\right]
$$

The total incident sound pressure $P$, the sound pressure $P_{D}$, and $P_{0}$ in the cavity all can be obtained once the response of the MPP and backing panel is solved. Then, the response of the whole system can be obtained.

2.2. Optimization of the Point Force. It should be noticed that (42) still contains the unknown variable of the control force amplitude $F_{s}$. The theoretically optimal objective function for the active MPPA is the sound absorption coefficient. It is an implicit function of the amplitude of the control force, which can be expressed as 


$$
\alpha=\frac{\Pi_{\mathrm{abs}}}{\Pi_{\mathrm{inc}}}=\frac{\rho_{0} c_{0} \int_{S} \operatorname{Re}\left[P(x, y)^{*} \cdot v_{1}(x, y)\right] \mathrm{d} x \mathrm{~d} y}{\left|p_{i}\right|^{2} S_{\mathrm{MPP}}} \propto F_{s},
$$

where $\Pi_{\mathrm{inc}}$ and $\Pi_{\mathrm{abs}}$ are incident and absorbed sound power, and $S_{\mathrm{MPP}}$ is the area of the MPP. For the above-complicated expression, the genetic algorithm (GA) can be applied to obtain the optimal amplitude of the control force for maximizing the sound absorption coefficient. Then, the response of the whole system in the controlled condition can be obtained accordingly.

\section{Active Control Results and Surface Impedance Analysis}

3.1. Parameter Assignment and Model Validation. The geometric and material parameters of the model used in the simulation are listed in Table 1. It is assumed that the material of the MPP and backing panel is aluminum. The dimension of the MPP is assigned with different values to verify the active control effect of the error sensing strategy in different cases. The secondary point force is located at $(0.05,0.05)$ so as to excite most of the modes of the backing panel contained in the low-frequency range. After a careful convergence study, the upper limit numbers of the cavity mode $(U$ and $W$ ) and the panel mode (the number of the MPP and backing panel mode is the same) are chosen as $U=W=9$ and $M=N=20$. The increment of the system response in uncontrolled conditions (such as the sound pressure at any position of the cavity) at the upper limit frequency of $1000 \mathrm{~Hz}$ is less than $1 \mathrm{~dB}$ when the numbers of these modes further increase, which guarantees the accuracy of the results. The modulus of the amplitude of the control force is set as $0<\left|F_{s}\right| \leq 1.5(N)$ when it is optimized by a genetic algorithm. This range is appropriate for the model considered in this research.

In order to validate the theoretical model, the active MPPA before control is also modeled by the finite element software COMSOL. The length and width of the MPP are $0.2 \mathrm{~m}$ and $0.3 \mathrm{~m}$. Similar to the theoretical model, the active MPPA is also placed at one end of a rectangular duct in the finite element model. The duct on the incident side is divided into two sections. The first section uses a perfectly matched layer (PML) to simulate the transmission of the reflected wave to the reverse infinity. The secondary section simulates the sound field of the incident plane wave. The MPP used in the model is set as the equivalent impedance surface already built in the COMSOL. The schematic diagram of the finite element model is shown in Figure 2. The sound absorption coefficient calculated by analytical and numerical methods is shown in Figure 3. The result of the MPPA with a rigid backing wall is also included. General good agreement is found among them, which validates the accuracy of the theoretical model. The elastic effect of the MPP is not considered in the finite element model. The MPP is also set to be rigid in the theoretical model. Compared with the traditional MPPA, the sound absorption coefficient of the MPPA with elastic backing panel generates two absorption peaks in the low frequency. This is due to the resonant vibration of the backing panel (the $(1,1)$ and $(1,3)$ mode with resonant frequencies $88 \mathrm{~Hz}$ and $306 \mathrm{~Hz}$ ) excited by the plane wave excitation, which can highly absorb and dissipate incident sound energy.

3.2. Active Control Results. The resonant frequency of the traditional MPPA with a rigid backing wall for the parameters used is $709 \mathrm{~Hz}$. Hence, the upper limit frequency considered in the active control is set as $700 \mathrm{~Hz}$. The cost function is the theoretically optimal control objective, i.e., the sound absorption coefficient of the active MPPA. The panel dimension is set as $0.2 \mathrm{~m} \times 0.3 \mathrm{~m}$. The sound absorption coefficient of the active MPPA before and after control is shown in Figure 4. It is highly improved after control in the low-frequency range, which demonstrates the feasibility of this approach. There are still small frequency bands in which the improvement of sound absorption is inapparent.

All other types of modes of the backing panel (except for the (odd, odd) mode) can be excited under the control of the point force. The backing panel radiates sound into the cavity and accordingly adjusts the particle velocity of the air media in the microporous and the velocity of the MPP. Then, the surface impedance of the active MPPA can be adjusted to match the characteristic impedance of the air. Hence, the sound absorption coefficient on the resonant frequencies of these exciting modes (such as $170 \mathrm{~Hz}, 354 \mathrm{~Hz}, 490 \mathrm{~Hz}$, $660 \mathrm{~Hz}$, and $680 \mathrm{~Hz},(1,2),(2,2),(2,3),(3,2)$, and $(2,4)$ mode) is highly improved after control. In fact, these modes can also be excited and play an important role in improving the sound absorption in a frequency band centered on each resonant frequency. Then, the total sound absorption improvement can be achieved in a wide successive frequency band. It should be noticed that the control effect on the resonant frequency of the $(2,1)$ mode $(272 \mathrm{~Hz})$ is not obvious. Accordingly, the frequency band of $250 \mathrm{~Hz}-300 \mathrm{~Hz}$ is also uncontrollable. It seems contradictory for the control results on the resonant frequencies of the $(1,2)$ and $(2,1)$ modes since the vibrational characteristic of these modes should be the same. The physical mechanism of such interesting phenomenon will not be involved in this research for reducing the length of the paper.

3.3. Surface Impedance of the Active MPPA. Unlike the onedimensional case, due to the complicated vibration of the backing panel, the surface impedance of the active MPPA varies over the surface. The sound absorption of the active MPPA exhibits obvious local characteristics [24-26]. The sound energy is absorbed in some areas of the surface but is reflected in other areas. The net sound energy absorption should be positive and will be highly improved in controlled conditions. This can be further illustrated by analyzing the change of the surface average impedance ratio $\left[\bar{Z}=\bar{P} /\left(\bar{v}_{1} \cdot \rho_{0} c_{0}\right)\right]$ of the active MPPA before and after control, which is shown in Figure 5.

The surface impedance of the active MPPA before control can be roughly considered as that of the MPPA with 
TABLE 1: The geometric and material parameters of the model.

\begin{tabular}{|c|c|c|c|}
\hline Parameter & Value & Parameter & Value \\
\hline Pore diameter of the MPP & $0.4 \times 10^{-3} \mathrm{~m}$ & Thickness of the backing panel & $0.001 \mathrm{~m}$ \\
\hline Thickness of the MPP & $0.5 \times 10^{-3} \mathrm{~m}$ & Density of aluminum & $2790 \mathrm{Kg} / \mathrm{m}^{3}$ \\
\hline Porosity of the MPP & $1 \%$ & Young's modulus of aluminum & $7.2 \times 10^{10} \mathrm{~N} / \mathrm{m}^{2}$ \\
\hline Cavity depth of the MPPA & $0.05 \mathrm{~m}$ & Poisson's ratio of aluminum & 0.34 \\
\hline Viscosity coefficient of air & $1.882 \times 10^{-5} \mathrm{~Pa} \cdot \mathrm{s}$ & Modal damping ratio of aluminum & 0.005 \\
\hline Density and sound speed of air & $\rho_{0}=1.21 \mathrm{Kg} / \mathrm{m}^{3} c_{0}=344 \mathrm{~m} / \mathrm{s}$ & The amplitude of the incident plane wave & $p_{0}=1 \mathrm{~Pa}$ \\
\hline
\end{tabular}

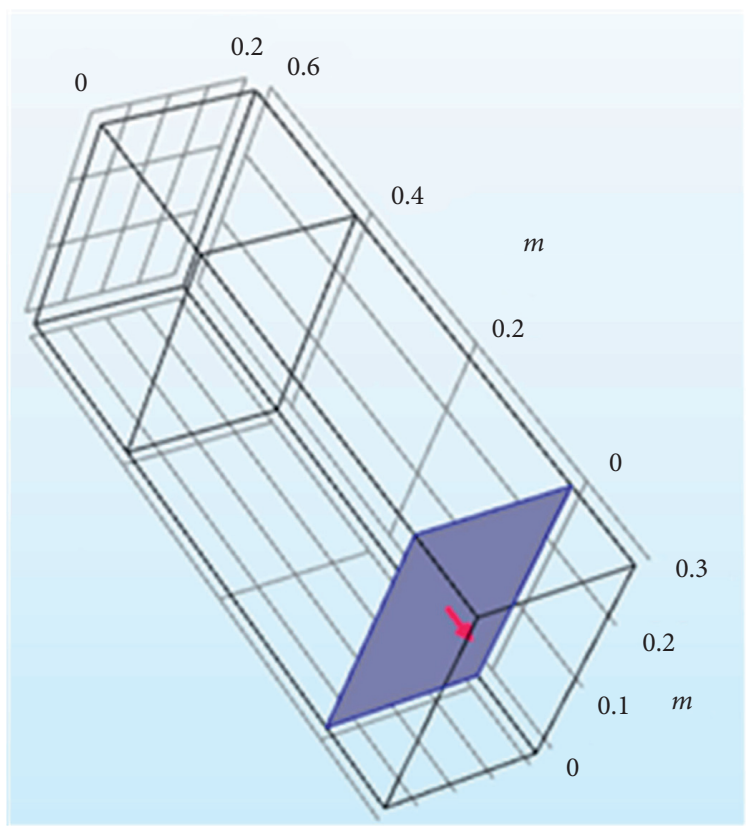

Figure 2: The schematic diagram of the finite element model.

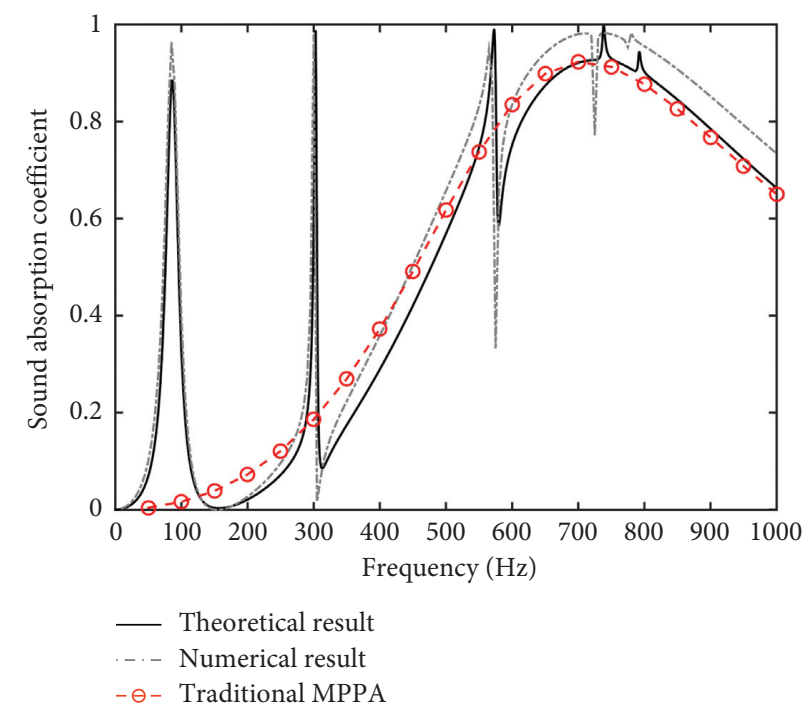

Figure 3: Comparison between the theoretical and numerical results.

a rigid wall if the backing panel is not highly excited on the resonant frequency. The average sound resistance is small and the average sound reactance is large in the low- frequency range, as shown in Figure 5. On some resonant frequencies of the MPP or backing panel, due to the vibro-acoustic coupling effect of the MPP-cavity-backing 


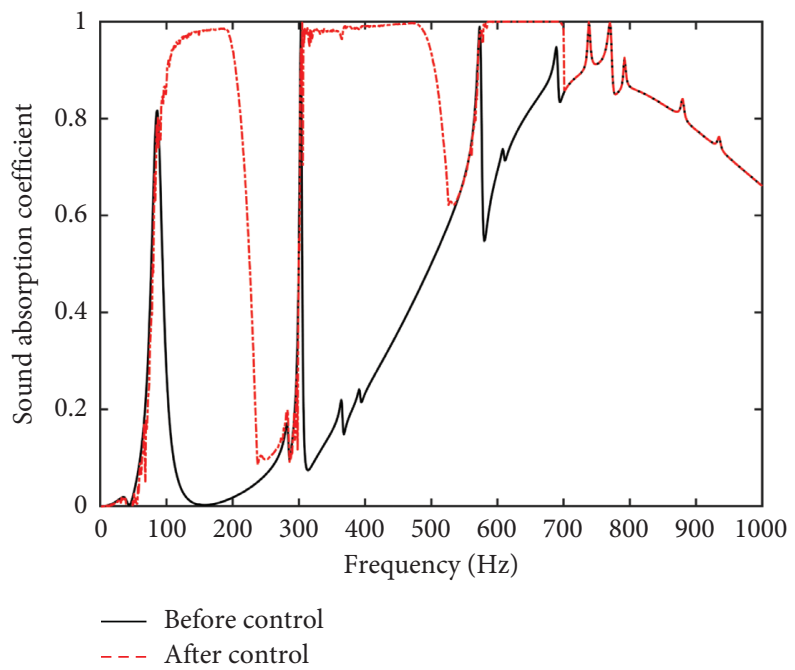

FIgURE 4: Sound absorption coefficient of the active MPPA before and after control.

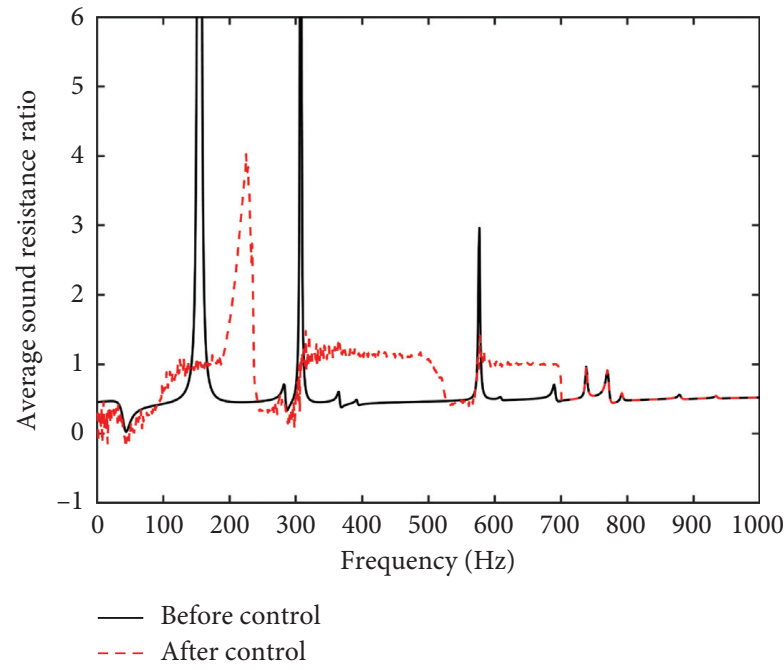

(a)

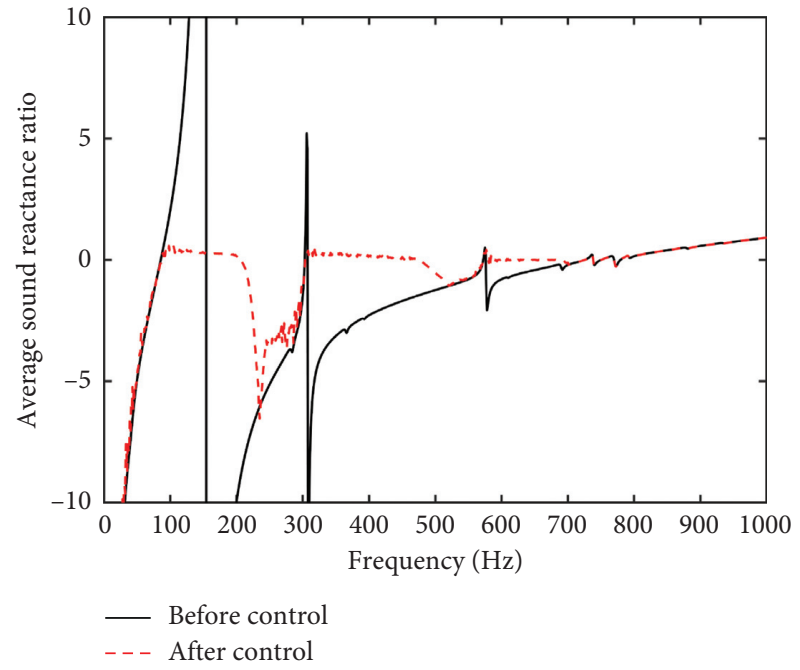

(b)

FIGURE 5: Surface average impedance ratio of the active MPPA before and after control: (a) sound resistance ratio and (b) sound reactance ratio.

panel, the average surface impedance has maximum and minimum.

Other types of resonant modes will be excited under the control of point force. Then, the surface impedance of the active MPPA will be changed, and its average value is adjusted to be close to the characteristic impedance of the air in the controllable frequency bands (the average resistance is close to $\rho_{0} c_{0}$, and the average reactance is close to 0 ), such as $100-250 \mathrm{~Hz}, 300-500 \mathrm{~Hz}$, and $600-700 \mathrm{~Hz}$. Hence, the total net absorbed sound energy is highly improved in these bands.

\section{Error Sensing Strategy of the Active MPPA}

4.1. Selection of the Objective Function. The optimal objective function in theory for the active MPPA is the sound absorption coefficient. The improvement of the sound absorption is maximal and the control effect is optimal in this case, which is called the optimal control state. However, the sound absorption coefficient cannot be measured directly in practice and can not be used as the cost function or error signal to feedback to the adaptive controller for iteratively calculating the optimal control force. Hence, selecting the measurable error signal that is highly correlated with the objective function of the sound absorption coefficient is a key factor for system implementation. For a one-dimensional sound field, the sound pressure of the plane wave in the cavity is used as the cost function for the PR strategy, which can be measured by one microphone and be canceled to adjust the surface impedance of the active MPPA. The reflected plane wave in the cavity is used as the cost function for the IM strategy, which can be measured by two microphones and also be canceled to adjust the surface impedance.

Due to the complicated vibration of the backing panel, the cavity sound field of the proposed active MPPA will be 
complicated. The above two strategies are not suitable for this case. It is obvious that the surface average impedance ratio of the active MPPA is highly correlated with the sound absorption coefficient. The closer the average impedance ratio is to 1 , the greater the sound absorption coefficient is. The surface average impedance ratio can be measured by arranging multiple acoustic vector sensors (AVSs, simultaneously measuring the sound pressure and particle velocity) on the surface of the active MPPA. The required number of the AVS depends on the complexity of the distribution of the surface impedance.

In order to further analyze the surface impedance of the active MPPA, the distribution of the surface resistance and reactance ratio on resonant frequencies of the $(1,2),(2,2)$, $(2,3)$, and $(3,2)$ modes after control is plotted in Figures 6-9 . It can be found that, due to the antisymmetric property of these resonant modes, the surface resistance and reactance of the active MPPA also approximately have the symmetric or antisymmetric properties in controlled conditions. The surface sound resistance is positive on one part of the surface and is negative on the other part. The sound resistance on the positive part is larger than the value on the negative part, which means that the sound energy absorption on the positive part is larger than the sound energy reflection on the negative part. The net sound energy absorption is highly improved after control and the average sound impedance of the active MPPA is close to the impedance of the air (such as in Figure 6(a)). This is the essential control mechanism of such active MPPA. Due to the symmetric or antisymmetric properties, the average surface impedance ratio in controlled conditions can be measured by using the limited number of the AVS. Provided these AVSs are arranged symmetrically, the surface average impedance ratio can be roughly predicted (for instance, only two AVSs are needed for the $(1,2)$ mode).

Based on the above analysis, after the surface average impedance ratio of the active MPPA is roughly measured, the following cost function can be constructed:

$$
J=\left|\frac{P_{1}+P_{2}+\cdots+P_{e}}{\left(v_{1,1}+v_{1,2}+\cdots+v_{1, e}\right) \rho_{0} c_{0}}-1\right| \propto F_{s},
$$

where $P_{1}, P_{2}$, and $P_{e}$ are the sound pressure on the sensing point, and $v_{1,1}, v_{1,2}$, and $v_{1, e}$ are the particle velocity. $\bar{Z}=$ $\bar{P} /\left(\bar{v}_{1} \cdot \rho_{0} c_{0}\right)=\left(P_{1}+P_{2}+\cdots+P_{e}\right) /\left(\left(v_{1,1}+v_{1,2}+\cdots+v_{1, e}\right)\right.$ $\left.\rho_{0} c_{0}\right)$ is the measured surface average impedance ratio. The smaller the cost function is, the closer the surface average impedance ratio is to 1 . When the surface average impedance ratio is close to 1 , the sound absorption of the active MPPA will achieve the maximum. Besides, the cost function $J$ is measurable since the surface average impedance ratio is measurable. Thus, it is an appropriate cost function to be used in practice. Theoretically, the genetic algorithm (GA) is applied to find the optimal control force by minimizing the cost function $J$. In practice, the optimal control signal for driving the control source can be iteratively calculated by using the Fx-LMS algorithm when minimizing the error signal $J$
4.2. Control Result Analysis. For the case of panel dimension being $0.2 \mathrm{~m} \times 0.3 \mathrm{~m}$, the maximum modal index is 3 in the low-frequency range $0-700 \mathrm{~Hz}$. Thus, eight VASs are used for constructing the cost function. The arrangement of the VAS on the surface of the active MPPA is shown in Figure 10. The active control result with (44) as the cost function is compared with the theoretically optimal result (when the sound absorption coefficient is used as the cost function) in Figure 11. It can be found that the control result for the proposed error sensing strategy is roughly consistent with the theoretical optimal result, which demonstrates the feasibility of the proposed method. Besides, except for the above resonant frequencies, it can also gain a good control effect on the off-resonant frequency of the backing panel.

In general, the more the number of sensing points, the closer the predictive value of the surface average impedance ratio is to the actual value. There needs a small number of the VAS for the small-sized active MPPA since the number of the mode of the backing panel in the low-frequency range is small. On the contrary, the backing panel contains a large number of modes in the low-frequency range for the largesized active MPPA, and it will need more VASs. Eight evenly arranged VASs are enough for roughly predicting the surface average impedance ratio of the active MPPA for the panel dimension being $0.2 \mathrm{~m} \times 0.3 \mathrm{~m}$. In order to further validate the effectiveness of this error sensing strategy, the above eight evenly arranged VASs are also used for the case of panel dimensions being $0.2 \mathrm{~m} \times 0.2 \mathrm{~m}$ and $0.3 \mathrm{~m} \times 0.3 \mathrm{~m}$. The active control results are shown in Figures 12 and 13. For the small-sized panel $(0.2 \mathrm{~m} \times 0.2 \mathrm{~m})$, eight VASs are enough for accurately predicting the average surface impedance, and the active control result is perfect. The sound absorption coefficient after control is also almost consistent with that of the optimal control state for the large-sized panel $(0.3 \mathrm{~m} \times 0.3 \mathrm{~m})$, which further validates the feasibility of the method.

It should be noticed that the sound absorption coefficient decreases on the uncontrollable frequency bands after control, especially on the frequency band $230-300 \mathrm{~Hz}$ in the case of panel dimension being $0.2 \mathrm{~m} \times 0.3 \mathrm{~m}$ and $200-250 \mathrm{~Hz}$ for the panel dimension being $0.3 \mathrm{~m} \times 0.3 \mathrm{~m}$. For the panel dimension being $0.2 \mathrm{~m} \times 0.3 \mathrm{~m}$, the sound absorption on the resonant frequency of $(2,1)$ mode is uncontrollable, which results in the above frequency band being uncontrollable. The sound resistance and reactance ratio of the active MPPA on the resonant frequency of the $(2,1)$ mode in the controlled condition is shown in Figure 14. It can be found that the distribution of the surface sound resistance is not symmetrical and also nonuniform on the positive half. Hence, only using eight VASs can not accurately predict the average surface sound impedance, which results in the fact that the cost function is not highly correlated with the sound absorption coefficient of the active MPPA. This leads to the fact that the sound absorption in the controlled condition on $230-300 \mathrm{~Hz}$ decreases after control. However, this has little influence on the total control effect since this frequency band is uncontrollable. 


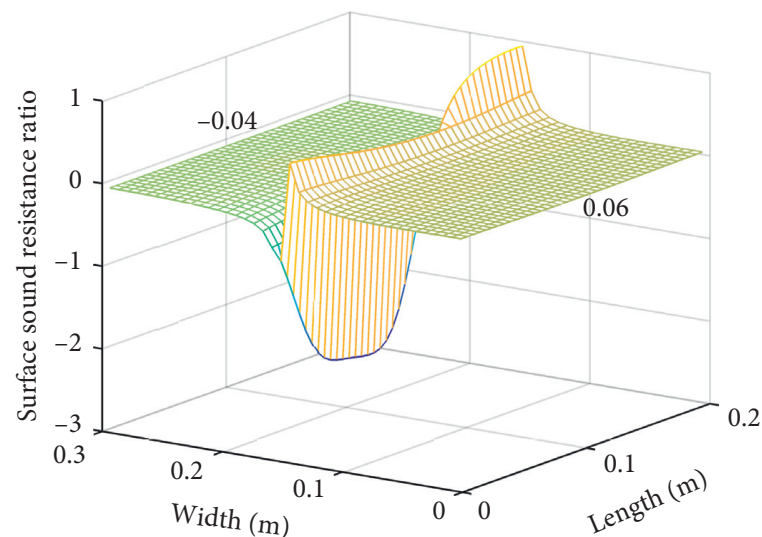

(a)

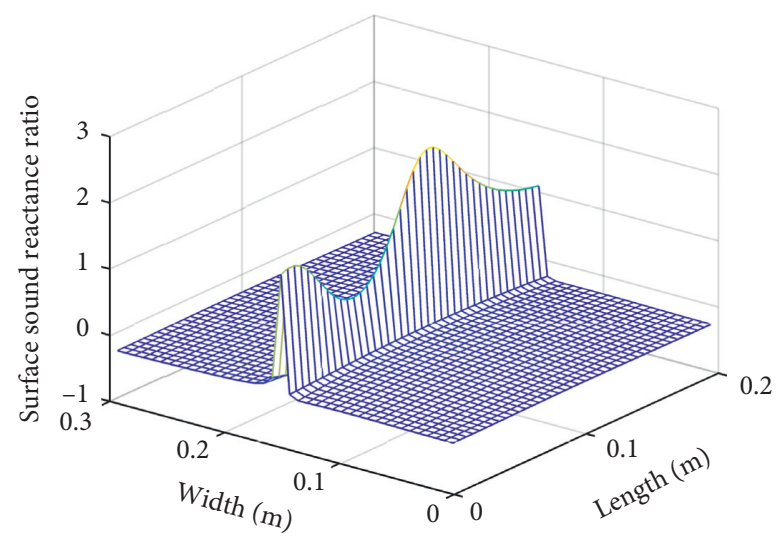

(b)

Figure 6: Surface sound impedance ratio of the active MPPA on the resonant frequency of the $(1,2)$ mode after control: (a) sound resistance ratio and (b) sound reactance ratio.

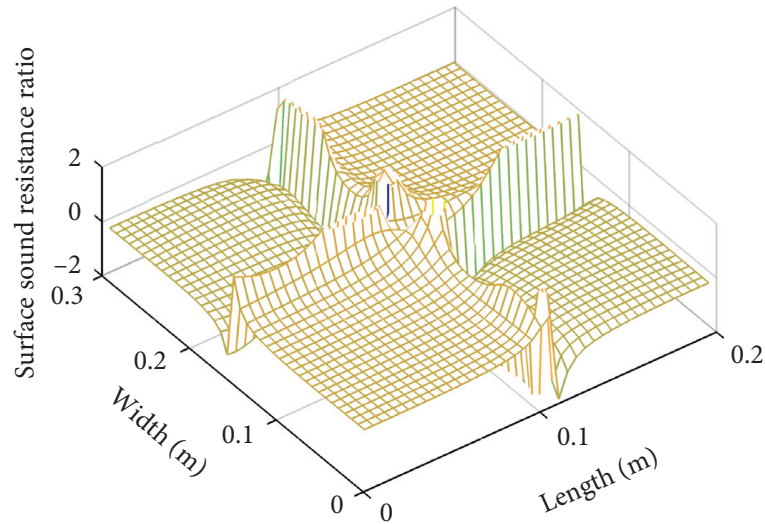

(a)

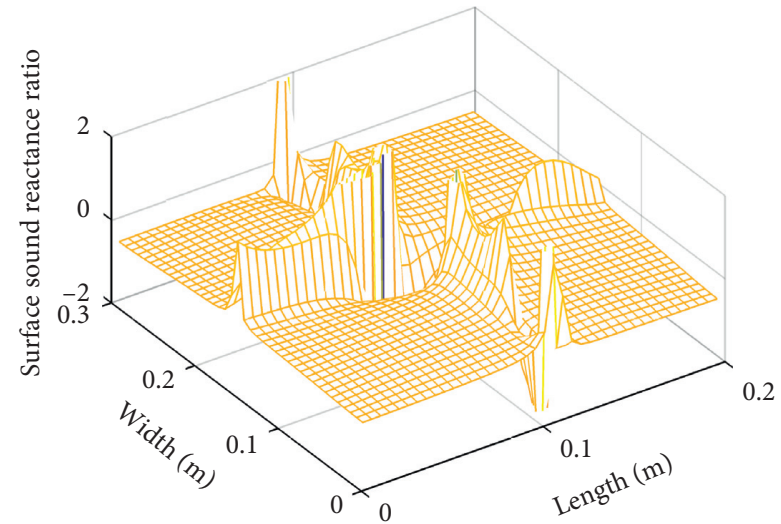

(b)

Figure 7: Surface sound impedance ratio of the active MPPA on the resonant frequency of the $(2,2)$ mode after control: (a) sound resistance ratio and (b) sound reactance ratio.

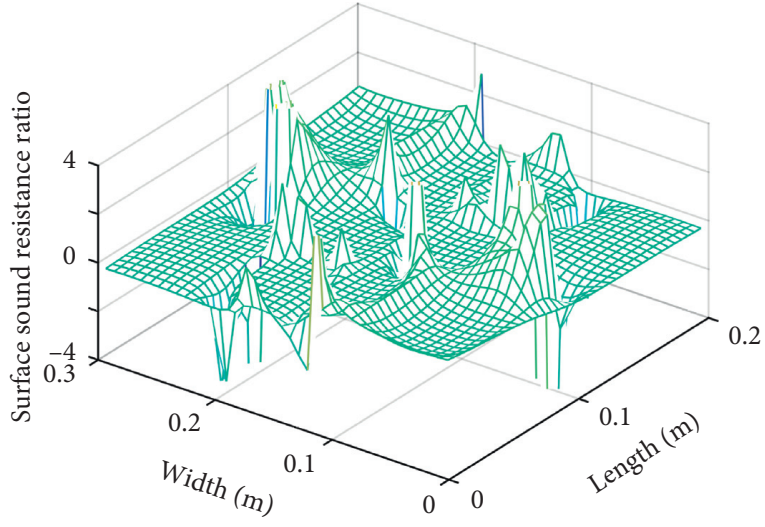

(a)

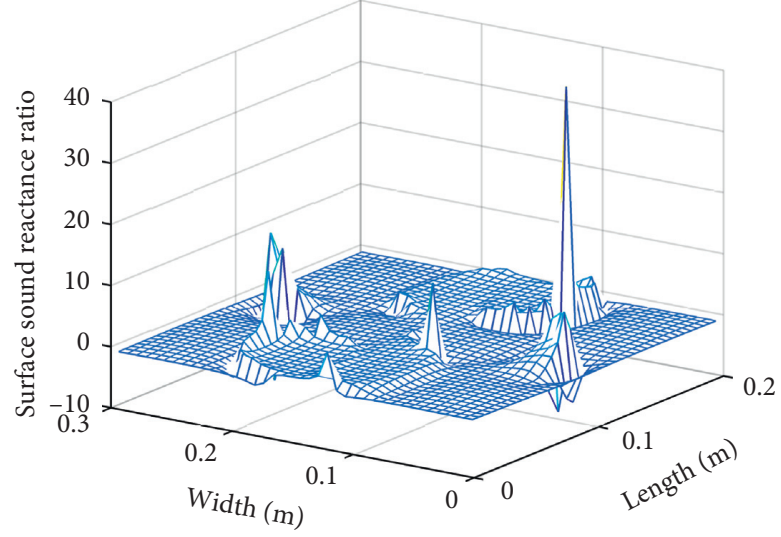

(b)

FIGURE 8: Surface sound impedance ratio of the active MPPA on the resonant frequency of the $(2,3)$ mode after control: (a) sound resistance ratio and (b) sound reactance ratio. 


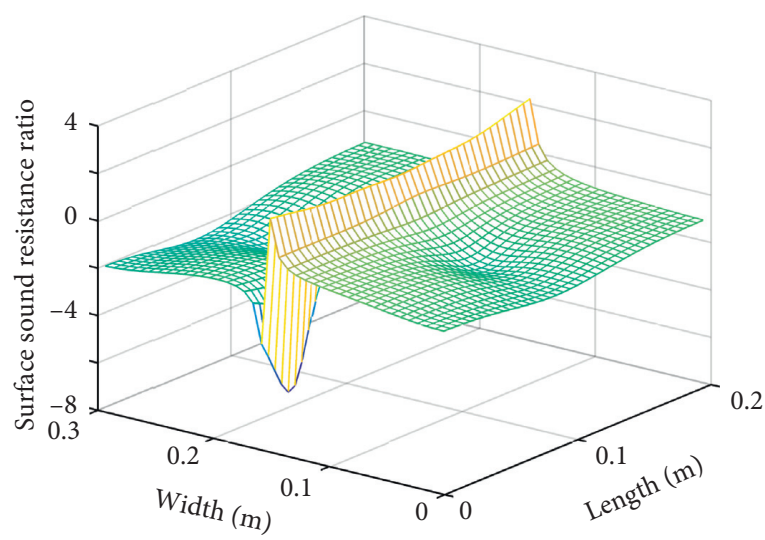

(a)

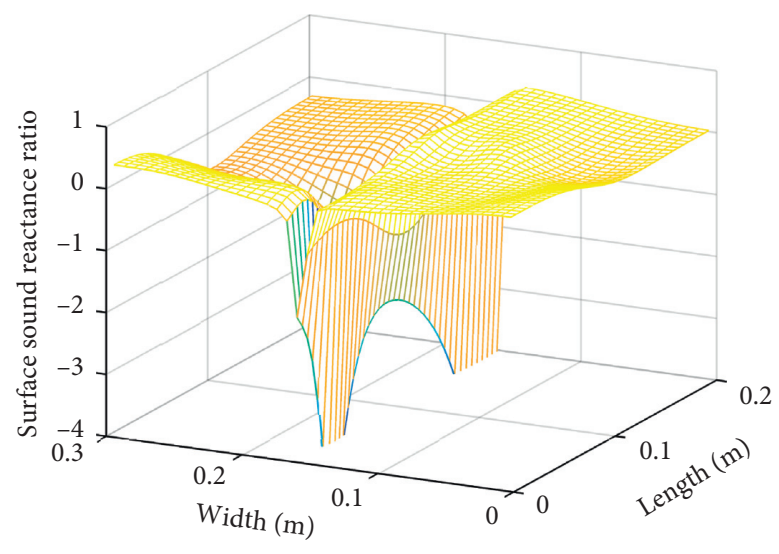

(b)

Figure 9: Surface sound impedance ratio of the active MPPA on the resonant frequency of the $(3,2)$ mode after control: (a) sound resistance ratio and (b) sound reactance ratio.

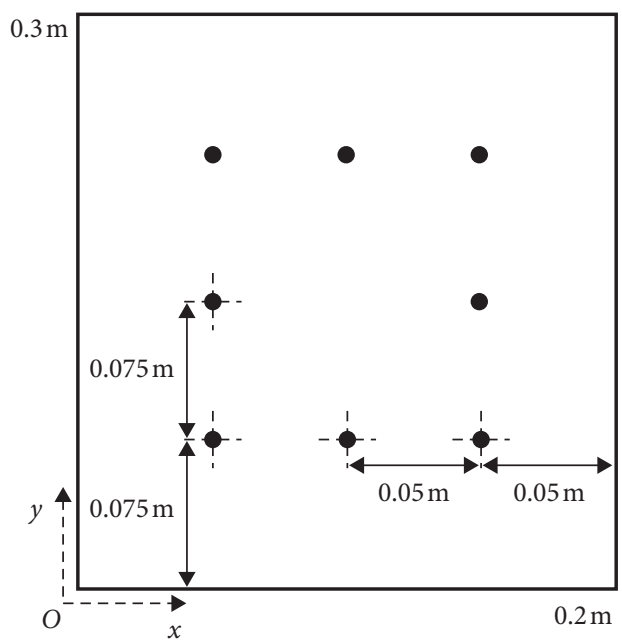

Figure 10: The arrangement of the VAS on the surface of the active MPPA.

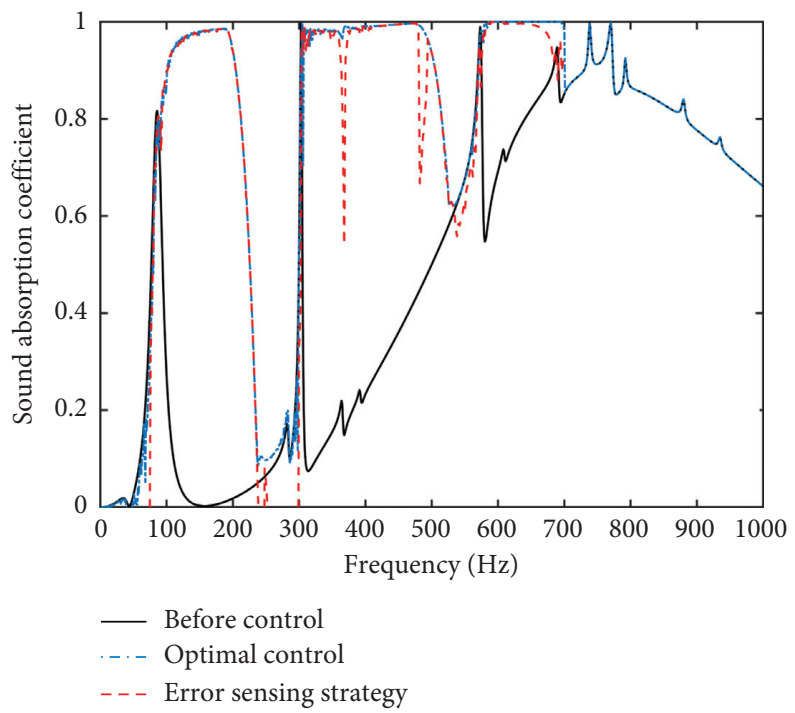

FIgURE 11: Active control results for the case of panel dimension being $0.2 \mathrm{~m} \times 0.3 \mathrm{~m}$. 


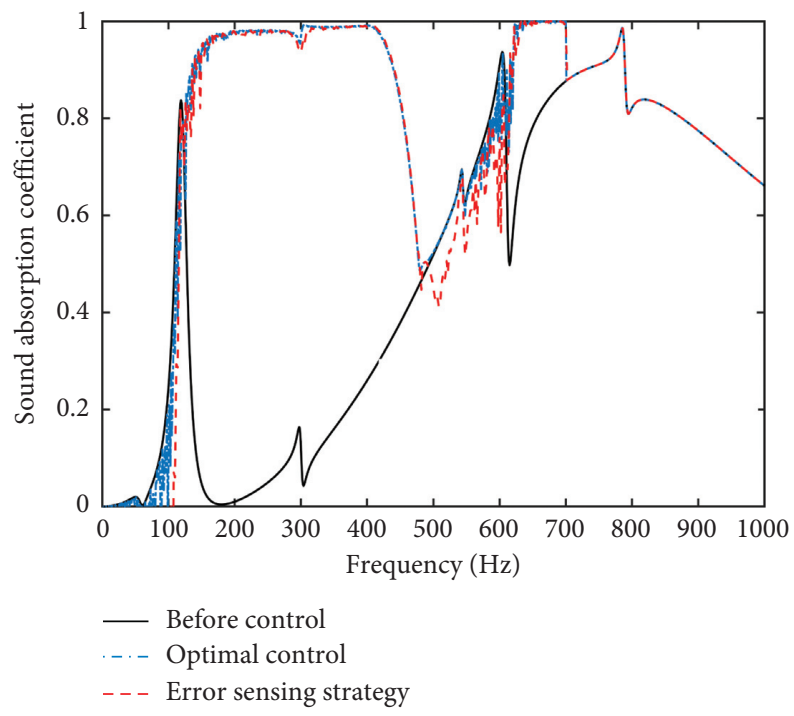

Figure 12: Active control results for the case of panel dimension being $0.2 \mathrm{~m} \times 0.2 \mathrm{~m}$.

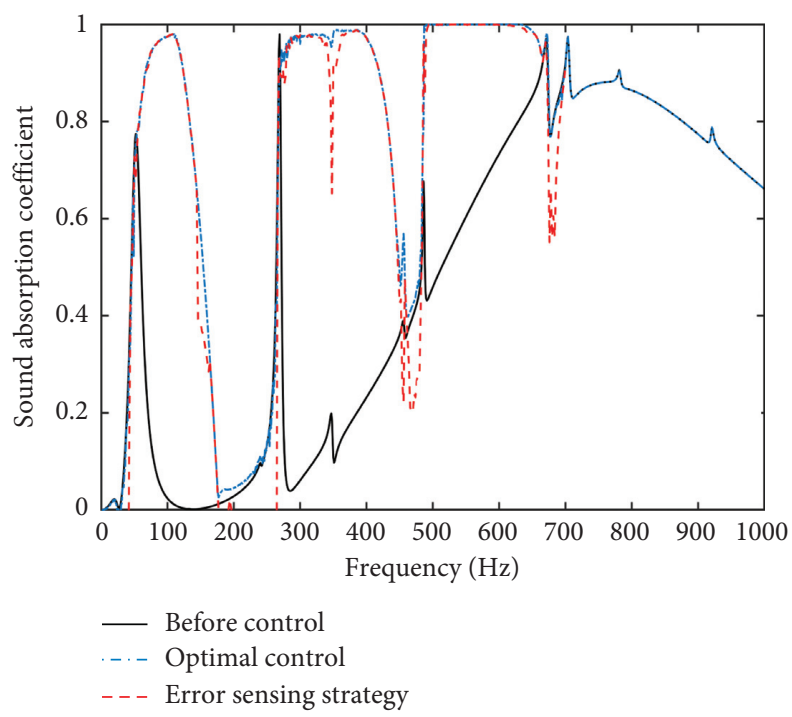

FIgURE 13: Active control results for the case of panel dimension being $0.3 \mathrm{~m} \times 0.3 \mathrm{~m}$.

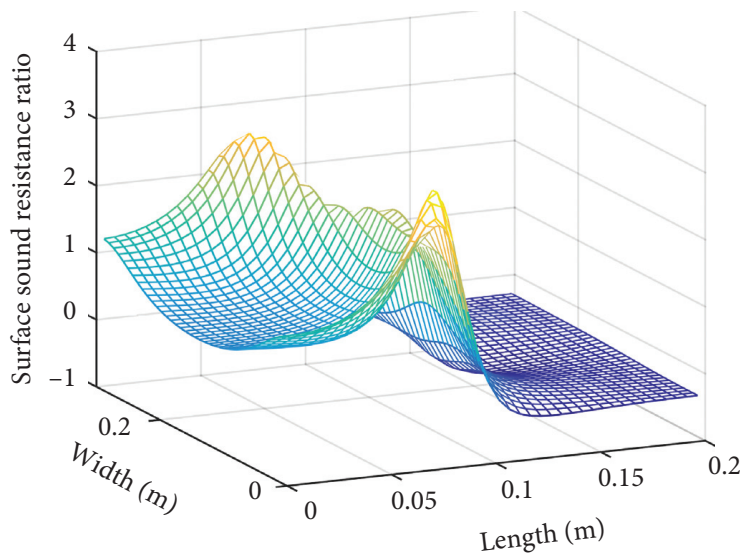

(a)

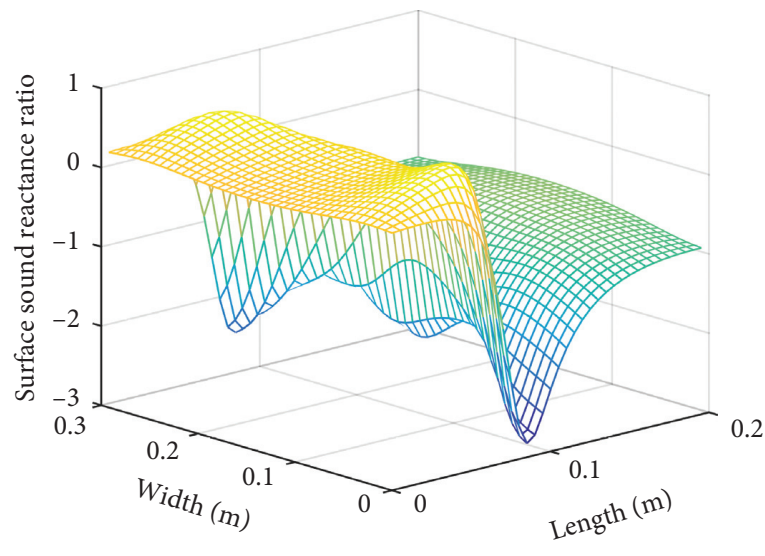

(b)

FIGURE 14: Surface sound impedance of the active MPPA on the resonant frequency of the $(2,1)$ mode after control: (a) sound resistance ratio and (b) sound reactance ratio. 


\section{Conclusions}

The error sensing strategy of the proposed active MPPA that uses the point force-controlled thin plate as the control source is constructed in this research. The surface average impedance of the active MPPA is adjusted to be close to the characteristic impedance of the air by the vibration of the backing panel in the controllable frequency bands. Due to the symmetric or antisymmetric properties of the surface impedance, the surface average impedance ratio can be roughly measured by using the limited number of the VAS. This measurable value is highly correlated with the sound absorption coefficient of the active MPPA and can be used to construct the cost function. Simulation results demonstrate that the active control result of the proposed error sensing strategy is nearly consistent with the theoretically optimal control result, which validates the effectiveness of this method.

\section{Data Availability}

The data supporting the research of the paper are available from the corresponding author upon request.

\section{Conflicts of Interest}

The authors declare that there are no conflicts of interest regarding the publication of this paper.

\section{Acknowledgments}

This work was financially supported by the China Postdoctoral Science Foundation (Grant no. 2019M663821), the Fundamental Research Funds for the Central Universities (Grant no. 3102019HHZY030025), the Natural Science Basic Research Plan in Shaanxi Province of China (Grant no. 2018JQ1025), and the National Natural Science Foundation of China (NSFC, Grant no. 51705421).

\section{References}

[1] X. Yu, L. Cheng, and X. You, "Hybrid silencers with microperforated panels and internal partitions," Journal of the Acoustical Society of America, vol. 137, no. 2, pp. 951-962, 2015.

[2] D. Maa, "Potential of microperforated panel absorber," Journal of the Acoustical Society of America, vol. 104, no. 5, pp. 2861-2866, 1998.

[3] H. Ruiz, P. Cobo, and F. Jacobsen, "Optimization of multiplelayer microperforated panels by simulated annealing," Applied Acoustics, vol. 72, pp. 772-776, 2011.

[4] K. Sakagami, T. Nakamori, M. Morimoto, and M. Yairi, "Double-leaf microperforated panel space absorbers: a revised theory and detailed analysis," Applied Acoustics, vol. 70, pp. 703-709, 2009.

[5] D. H. Lee and Y. P. Kwon, "Estimation of the absorption performance of multiple layer perforated panel systems by transfer matrix method," Journal of Sound and Vibration, vol. 278, pp. 847-860, 2004.

[6] C. Wang and L. Huang, "On the acoustic properties of parallel arrangement of multiple micro-perforated panel absorbers with different cavity depths," Journal of the Acoustical Society of America, vol. 130, no. 1, pp. 208-218, 2011.

[7] C. Wang, L. Huang, and Y. Zhang, "Oblique incidence sound absorption of parallel arrangement of multiple micro-perforated panel absorbers in a periodic pattern," Journal of Sound and Vibration, vol. 333, pp. 6828-6842, 2014.

[8] C. Q. Wang, L. Cheng, J. Pan, and G. H. Yu, "Sound absorption of a micro-perforated panel backed by an irregularshaped cavity," Journal of the Acoustical Society of America, vol. 127, no. 1, pp. 238-246, 2010.

[9] C. Yang, L. Cheng, and J. Pan, "Absorption of oblique incidence sound by a finite micro-perforated panel absorber," Journal of the Acoustical Society of America, vol. 133, no. 1, pp. 201-209, 2013.

[10] T. Bravo and C. Maury, "Sound attenuation and absorption by micro-perforated panels backed by anisotropic fibrous materials: theoretical and experimental study," Journal of Sound and Vibration, vol. 425, pp. 189-207, 2018.

[11] Y. J. Qian, D. Y. Kong, S. M. Liu, S. M. Sun, and Z. Zhao, "Investigation on micro-perforated panel absorber with ultramicroperforations," Applied Acoustics, vol. 74, pp. 931-935, 2013.

[12] X. Gai, X. Li, B. Zhang, T. Xing, J. Zhao, and Z. Ma, "Experimental study on sound absorption performance of microperforated panel with membrane cell," Applied Acoustics, vol. 110, pp. 241-247, 2016.

[13] X. Gai, T. Xing, X. Li, and B. Zhang, "Sound absorption properties of microperforated panel with membrane cell and mass blocks composite structure," Applied Acoustics, vol. 137, pp. 98-107, 2018.

[14] X. Gai, T. Xing, X. Li, B. Zhang, and W. Wang, "Sound absorption of microperforated panel mounted with Helmholtz resonators," Applied Acoustics, vol. 114, pp. 260-265, 2016.

[15] S. Park, "Acoustic properties of micro-perforated panel absorbers backed by Helmholtz resonators for the improvement of low-frequency sound absorption," Journal of Sound and Vibration, vol. 332, pp. 4895-4911, 2013.

[16] X. H. Duan, H. Q. Wang, Z. B. Li et al., "Sound absorption of a flexible micro-perforated panel absorber based on PVDF piezoelectric film," Applied Acoustics, vol. 88, pp. 84-89, 2015.

[17] J. Tao, R. Jing, and X. Qiu, "Sound absorption of a finite micro-perforated panel backed by a shunted loudspeaker," Journal of the Acoustical Society of America, vol. 135, no. 1, pp. 231-238, 2014.

[18] X. Zhao and X. Fan, "Enhancing low frequency sound absorption of micro-perforated panel absorbers by using mechanical impedance plates," Applied Acoustics, vol. 88, pp. 123-128, 2015.

[19] P. Cobo, J. Pfretzschner, M. Cuesta, and D. K. Anthony, "Hybrid passive-active absorption using microperforated panels," Journal of the Acoustical Society of America, vol. 116, no. 4, pp. 2118-2125, 2004.

[20] P. Cobo and M. Cuesta, "Hybrid passive-active absorption of a microperforated panel in free filed conditions," Journal of the Acoustical Society of America, vol. 121, pp. EL251-EL255, 2007.

[21] P. Cobo and M. Cuesta, "Measuring hybrid passive-active sound absorption of a microperforated liner at oblique incidence," Journal of the Acoustical Society of America, vol. 125, no. 1, pp. 185-190, 2009.

[22] A. J. Pretlove, "Free vibrations of a rectangular panel backed by a closed rectangular cavity," Journal of Sound and Vibration, vol. 2, pp. 197-209, 1965. 
[23] Y. Y. Lee, E. W. M. Lee, and C. F. Ng, "Sound absorption of a finite flexible micro-perforated panel backed by an air cavity," Journal of Sound and Vibration, vol. 287, pp. 227-243, 2005.

[24] T. Bravo, C. Maury, and C. Pinhede, "Vibroacoustic properties of thin micro-perforated panel absorbers," Journal of the Acoustical Society of America, vol. 132, no. 2, pp. 789-798, 2012.

[25] T. Bravo, C. Maury, and C. Pinhede, "Sound absorption and transmission through flexible micro-perforated panels backed by an air layer and a thin plate," Journal of the Acoustical Society of America, vol. 131, no. 5, pp. 3853-3863, 2012.

[26] T. Bravo, C. Maury, and C. Pinhede, "Enhancing sound absorption and transmission through flexible multi-layer micro-perforated structures," Journal of the Acoustical Society of America, vol. 134, no. 5, pp. 3663-3673, 2013. 